\title{
Plane Detection Using Particle Swarm Optimization and Conformal Geometric Algebra
}

\author{
Carlos López-Franco ${ }^{1, \star}$, Jesús Hernández-Barragán ${ }^{1}$, Michel López-Franco $^{2}$, \\ Nancy Arana-Daniel ${ }^{1}$, and Alma Y. Alanís ${ }^{1}$ \\ 1 Computer Science Department, CUCEI, University of Guadalajara, Mexico \\ ${ }^{2}$ CINVESTAV Guadalajara, Mexico
}

\begin{abstract}
The detection of planes is a useful capability for a safe navigation algorithm, which is a crucial task for robots working in indoor environments. In this paper, the authors present an approach for plane detection based on Conformal Geometric Algebra, and Particle Swarm Optimization, with data obtained from an RGB-D sensor. The simulation and experimental results show the effectiveness of the proposed approach.
\end{abstract}

Keywords: Plane detection, Conformal Geometric Algebra, Particle Swarm Optimization (PSO).

\section{Introduction}

An essential task for mobile robots is to move safely in its environment. In human-made indoor environments, the detection of planes is a key problem. One interesting and promising framework for representing geometric objects and its operations is the Conformal Geometric Algebra (CGA) [8]. It also has been used for many different robotics task and computer vision problems. In this work we use CGA to represent the planes, since it will allow us to extend it to different geometric objects and robotics applications.

In the last years, stochastic optimization methods have become very popular, they have been successfully applied in many research and applications areas. In general there five popular algorithms: Genetic Algorithms 6], Memetic algorithms [9], Particle Swarm Optimization (PSO) is is an population based optimization [7, Ant-Colony optimization [1] and Shuffled frog leaping algorithm [3]. In [2], the authors present a comparison study of these five EA, they conclude that the PSO algorithm performs better in general, with respect to the quality of the solution and the success rate. For these reasons, we decided to choose the PSO algorithm among the others. To detect the planes we propose the use of PSO in conjunction with CGA.

Recently, the RGB-D sensors have gained the attention of researchers, sensors like the Primesense sensor or the Kinect camera, acquire RGB data as normal

* This work has been partially supported by the CONACYT projects CB-156567, CB-103191, CB-106838, and INFR-229696.

E. Bayro-Corrochano and E. Hancock (Eds.): CIARP 2014, LNCS 8827, pp. 852859 2014.

(C) Springer International Publishing Switzerland 2014 
camera and depth information simultaneously. These sensors are able to provide data for real-time applications. In this work we propose a plane detection algorithm based on PSO and CGA with data obtained from an RGB-D sensor.

The rest of the paper is organized as follows: The next section will present a brief introduction to Geometric Algebra and CGA. Then, in section 3 the PSO algorithm is described. In section 4, the proposed approach based on PSO and CGA is presented. In section 5, the simulations and experimental results are given. Finally, in section [6] we give the conclusions.

\section{Geometric Algebra}

Let $\mathcal{V}^{n}$ denote a $n$-dimensional real vector space, endowed with an inner product $\mathbf{u}_{1} \cdot \mathbf{u}_{2}$ for vectors $\mathbf{u}_{1}, \mathbf{u}_{2} \in \mathcal{V}$. The geometric algebra [5] $\mathcal{G}_{n}=\mathcal{G}(\mathcal{V})$ consists of all sums of products $\mathbf{u}_{1} \mathbf{u}_{2} \ldots \mathbf{u}_{k}$ of vectors $\mathbf{u}_{i} \in \mathcal{V}$ subject to the following two principles of geometric multiplication:

$-\mathbf{u}^{2}=\mathbf{u} \cdot \mathbf{u}$ for all $\mathbf{u} \in \mathcal{V}$

- If the vectors $\mathbf{u}_{i}$ are pairwise orthogonal $\left(\mathbf{u}_{i} \cdot \mathbf{u}_{j}=0\right)$, then the geometric product of $r$ orthogonal vectors $\mathbf{u}_{1} \mathbf{u}_{2} \ldots \mathbf{u}_{r}$ is skewsymmetric over the interchange of any pair of vectors, i.e. $\mathbf{u}_{i} \mathbf{u}_{j}=-\mathbf{u}_{j} \mathbf{u}_{i}$ for $i \neq j$.

The geometric product of vectors $\mathbf{a}$ and $\mathbf{b}$ can be decomposed into a

$$
\mathbf{a b}=\mathbf{a} \cdot \mathbf{b}+\mathbf{a} \wedge \mathbf{b} .
$$

where $\mathbf{a} \cdot \mathbf{b}$ and $\mathbf{a} \wedge \mathbf{b}$ represent the symmetric and antisymmetric parts of the geometric product respectively.

There is insufficient space here to provide a compressive introduction to geometric algebra, however there are many good references that are available [45].

\subsection{Conformal Geometric Algebra}

The Euclidean geometric algebra $\mathcal{G}_{n}$ is embedded into a higher dimensional space $\mathcal{G}_{n+1,1}$ called the conformal conformal geometric algebra (CGA) 8]. Thus, the $3 \mathrm{D}$ Euclidean space $\mathcal{G}_{3}$ is embedded into the conformal geometric algebra $\mathcal{G}_{4,1}$, which includes the Euclidean basis vectors $\mathbf{e}_{1}, \mathbf{e}_{2}, \mathbf{e}_{3}$ and two extra basis vectors denoted by $\mathbf{e}_{+}$and $\mathbf{e}_{-}$. These basis vectors satisfy $\mathbf{e}_{1}^{2}=\mathbf{e}_{2}^{2}=\mathbf{e}_{3}^{2}=\mathbf{e}_{+}^{2}=1$ and $\mathbf{e}_{-}^{2}=-1$. With the basis vectors $\mathbf{e}_{+}$and $\mathbf{e}_{-}$we define two null vectors

$$
\begin{aligned}
\mathbf{e}_{0} & =\frac{\mathbf{e}_{-}-\mathbf{e}_{+}}{2}, \\
\mathbf{e}_{\infty} & =\mathbf{e}_{-}+\mathbf{e}_{+}
\end{aligned}
$$

where the vector $\mathbf{e}_{0}$ is interpreted as the coordinate system origin, and the vector $\mathbf{e}_{\infty}$ as the point at infinity. 
The outer product of $\mathbf{e}_{+}$and $\mathbf{e}_{-}$produces a special bivector

$$
E=\mathbf{e}_{+} \mathbf{e}_{-}
$$

called the E-plane, which represents the Minkowski plane [5].

In conformal geometric algebra a point is defined as

$$
x=\mathbf{x}+\frac{1}{2} \mathbf{x}^{2} \mathbf{e}_{\infty}+\mathbf{e}_{0}
$$

where $\mathbf{x} \in \mathcal{G}_{3}$ represents the Euclidean point and $x \in \mathcal{G}_{4,1}$ represents the conformal point.

A conformal plane is defined as

$$
p=\mathbf{n}+\delta \mathbf{e}_{\infty}
$$

where $\mathbf{n} \in \mathcal{G}_{3}$ represents the plane normal, and where $\delta$ is the distance of the plane from the origin.

The distance from the plane $p$ to a point $x$ can be computed as

$$
\begin{aligned}
p \cdot x & =\left(\mathbf{n}+\delta \mathbf{e}_{\infty}\right) \cdot\left(\mathbf{x}+\frac{1}{2} \mathbf{x}^{2} \mathbf{e}_{\infty}+\mathbf{e}_{0}\right) \\
& =\mathbf{n} \cdot \mathbf{x}-\delta
\end{aligned}
$$

The plane can be computed with the outer product of three conformal points and the point at infinity, that is

$$
\begin{aligned}
\hat{p}^{*} & =\mathbf{e}_{\infty} \wedge x_{1} \wedge x_{2} \wedge x_{3} \\
& =\mathbf{e}_{\infty} \wedge \mathbf{x}_{1} \wedge \mathbf{x}_{2} \wedge \mathbf{x}_{3}+E\left(\mathbf{x}_{2}-\mathbf{x}_{1} \wedge\left(\mathbf{x}_{3}-\mathbf{x}_{1}\right)\right.
\end{aligned}
$$

The plane $\hat{p}^{*}$ is not normalized, it can be normalized as

$$
p^{*}=\frac{\hat{p}^{*}}{\left\|\hat{p}^{*}\right\|}
$$

The relationship between (6) and (10) is

$$
p=-\hat{p}^{*} I
$$

where $I$ represents the pseudoscalar of the algebra.

\section{Particle Swarm Optimization}

Particle swarm optimization is a population based optimization technique inspired by the social behavior of bird flock [7. The PSO algorithm starts with a population of particles whose positions represent the potential solutions for the given problem. The optimal solution is found by updating the particles positions in each generation, with 


$$
\mathbf{x}_{i}(k)=\mathbf{x}_{i}(k-1)+\mathbf{v}_{i}(k)
$$

where the velocities of the particles are

$$
\mathbf{v}_{i}(k)=\alpha \phi_{0} \mathbf{v}_{i}(k-1)+c \phi_{1}\left(\mathbf{p}_{i}-\mathbf{x}_{i}(k-1)\right)+c \phi_{2}\left(\mathbf{p}_{g}-\mathbf{x}_{i}(k-1)\right)
$$

where $k$ is the number of iteration, $\mathbf{x}_{i}$ is each one of the particles, $v_{i}$ the velocity of the particle, $\alpha$ is an inertia factor, it makes the particle to keep its direction if this value is big. The terms $\phi_{0}, \phi_{1}, \phi_{2}$ represent random values. The values $\mathbf{p}_{i}$ and $\mathbf{p}_{g}$ represent the best position of the particle and the best global position respectively, these values are determined by the evaluation of some defined fitness measure. A review of PSO algorithm and its modification can be found in $7[10$.

\section{Particle Swarm Optimization for Plane Detection}

As we know, PSO is designed for continuous spaces, so in order to use it on a RGB image, provided by the RGB-D sensor, we have to work in a continuous normalized plane and then transform these results into pixels to use them on the image, Fig. 11ilustrates this conversion.

The size of the image provided by the RGB-D sensor is $640 \times 480$ pixels. To change from the continuous plane to the image plane we use

$$
\begin{aligned}
& \rho_{x}=\operatorname{round}\left(320 \alpha_{x}-320\right) \\
& \rho_{y}=\operatorname{round}\left(240-240 \alpha_{y}\right)
\end{aligned}
$$

where $\alpha_{x}$ and $\alpha_{y}$ represent the coordinates of the particle $\mathbf{x}_{i}$, and where $\rho_{x}, \rho_{y}$ are the pixels coordinates.

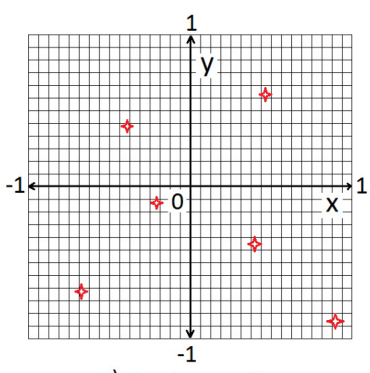

a) Continuous Plane

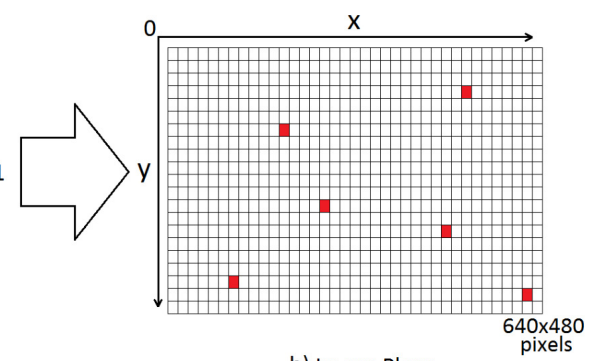

b) Image Plane

Fig. 1. Transformation from continuous plane to the image plane, a) PSO particles at some iteration b) Corresponding particles on the image plane 


\subsection{Description of the Method}

The algorithm start with the initialization of the particles with random values from -1 to 1 , so they are inside the normalized plane. Then for each particle $x_{i}$ we compute a candidate plane with the outer product of the pose of the particle and two neighbors using (6)-(10), which can be summarized as

$$
p_{i}=-\frac{\mathbf{e}_{\infty} \wedge x_{i-1} \wedge x_{i} \wedge x_{i+1}}{\left|\mathbf{e}_{\infty} \wedge x_{i-1} \wedge x_{i} \wedge x_{i+1}\right|} I
$$

With the candidate plane $p_{i}$ we compute the fitness value of the particle as

$$
\eta_{i}=\sum_{i=0}^{W} \sum_{j=0}^{H} f\left(p \cdot x_{i, j}\right)
$$

where $W$ and $H$ are the size of window around the particle, and where

$$
f(\alpha)= \begin{cases}1 & \text { if } \alpha<\epsilon \\ 0 & \text { otherwise }\end{cases}
$$

where $\epsilon$ is a threshold value. Then the local and global bests values are computed, and the algorithm is repeated until a stop criteria is met. The stop criteria can be defined as a maximum of iterations or a minimum of supporting plane points. The supporting points of a plane are the points that satisfy (18). A resume of the algorithm is shown in Algorithm 1 .

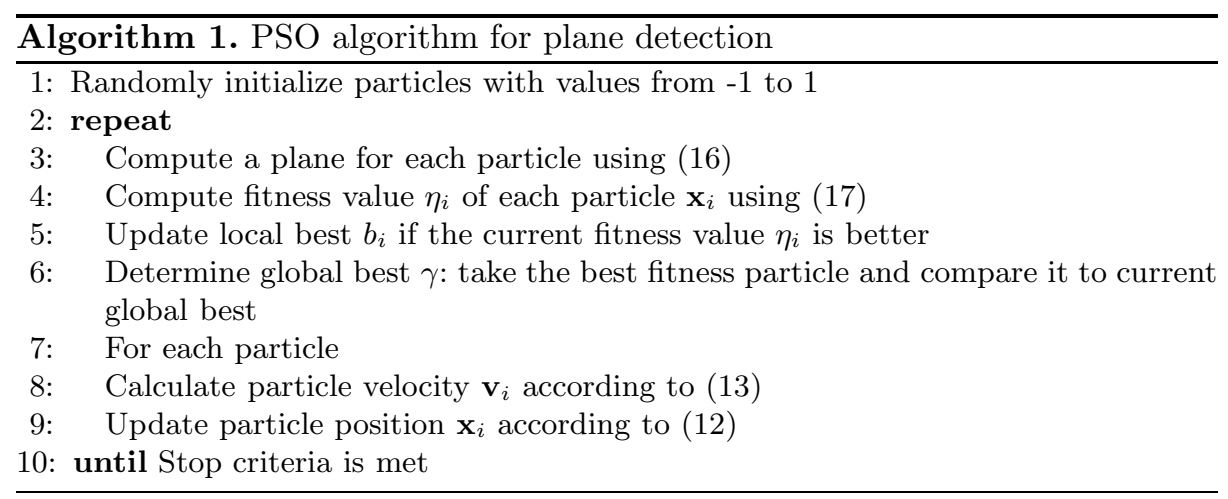

\section{Results}

To experimentally test the proposed approach, we performed simulations and real experiments. 


\subsection{Simulations Results}

The intention of the simulations experiments is to test the behavior of the proposed approach under controllable varying factors. The simulation is conducted as follow: first a 3D plane is defined, then its point cloud is computed. Then, in the first test we add different percentages of outliers. In the second experiments we test the proposed approach against different levels of noise. In the simulations, the reference plane is defined as $p=0.19183 \mathbf{e}_{1}+0.408380 \mathbf{e}_{2}+0.892928 \mathbf{e}_{3}+$ $2.13107 \mathbf{e}_{\infty}$

Outliers Test. In this experiment, we tested the proposed approach adding different percentages of outliers, varying from $10 \%$ to $90 \%$. The results are presented in Table 1. For every outlier percentage level, the algorithm runs 1, 000 times and output the estimated plane.

Table 1. Outlier test, the percentage of outliers goes from $10 \%$ to $90 \%$. The $\theta$ value, represents the angle between the normal of the reference plane and the normal of the detected plane. The value $D$ is the RMS of $d_{r}-d$, where $d_{r}$ and $d$ represent the distance of the plane from the origin for the reference plane and the estimated plane respectively.

\begin{tabular}{|c|c|c|c|c|c|c|c|}
\hline$\%$ outlier & time $(\mathrm{sec})$ & $\mathbf{e}_{1}$ & $\mathbf{e}_{2}$ & $\mathbf{e}_{3}$ & $\mathbf{e}_{\infty}$ & $\theta$ & $D$ \\
\hline 10 & 0.000200 & 0.191830 & 0.408380 & 0.892428 & 2.131070 & 0.000000 & 0.000003 \\
20 & 0.000400 & 0.191830 & 0.408380 & 0.892428 & 2.131070 & 0.000000 & 0.000003 \\
30 & 0.000400 & 0.191830 & 0.408380 & 0.892428 & 2.131070 & 0.000000 & 0.000003 \\
40 & 0.000600 & 0.191830 & 0.408380 & 0.892428 & 2.131070 & 0.000000 & 0.000003 \\
50 & 0.000800 & 0.191830 & 0.408380 & 0.892428 & 2.131070 & 0.000000 & 0.000003 \\
60 & 0.000800 & 0.191830 & 0.408380 & 0.892428 & 2.131070 & 0.000000 & 0.000003 \\
70 & 0.001000 & 0.191830 & 0.408380 & 0.892428 & 2.131070 & 0.000000 & 0.000003 \\
80 & 0.001200 & 0.191830 & 0.408380 & 0.892428 & 2.131070 & 0.000000 & 0.000003 \\
90 & 0.463600 & 0.191830 & 0.408380 & 0.892428 & 2.131070 & 0.000000 & 0.000003 \\
\hline
\end{tabular}

The results presented in Table 1 were obtained with a swarm size of 300 particles, and a maximum of 30 iterations. We can observe that the estimated plane is the same as the reference plane. Therefore, we can conclude that the proposed approach is very robust to outliers. With respect to the speed, we can notice that the proposes approach performs below 0.01 which can be enough for a real-time application. With respect to the $90 \%$ of outliers we can observe that the algorithm is slower with respect to the other levels, but this is due to the amount of effort required to find the plane.

\section{$5.2 \quad$ Noise Test}

The results presented in Table2 were obtained with a swarm size of 300 particles, and a maximum of 30 iterations. Different levels of noise were applied to the $3 D$ point cloud. For every noise level, the algorithm runs 1,000 times and output the mean angle between the reference plane and the estimates plane, and the RMS distance between the planes. 
From these results, we can notice that for large values of noise the depth is more affected that the normal of the plane. For noise levels lower than $10 \%$ the angle error is lower than $3.3 \mathrm{deg}$ and the distance between the planes is lower than $.12 \mathrm{~m}$. We have to notice that during the experiments with real data the noise found was lower than $10 \%$.

Table 2. Noise test, the percentage of noise goes from $5 \%$ to $50 \%$. The $\theta$ value, represents the mean angle between the normal of the reference plane and the normal of the detected plane. The value $D$ represent the RMS of $d_{r}-d$, where $d_{r}$ and $d$ are the distance of the plane from the origin for the reference plane and the estimated plane respectively.

\begin{tabular}{|c|c|c|c|}
\hline$\%$ noise & time $(\mathrm{sec})$ & $\theta$ & $\mathrm{D}$ \\
\hline 5 & 0.0006 & 1.8513 & 0.0521 \\
10 & 0.0002 & 3.2896 & 0.1102 \\
15 & 0.0022 & 9.5670 & 0.2424 \\
20 & 0.0036 & 10.6901 & 0.2479 \\
25 & 0.0024 & 9.3214 & 0.3117 \\
30 & 0.0028 & 9.0353 & 0.3792 \\
35 & 0.0244 & 9.2277 & 0.6573 \\
40 & 0.687 & 8.7435 & 0.8402 \\
45 & 0.6624 & 8.8801 & 1.0338 \\
50 & 0.7738 & 9.2361 & 1.2513 \\
\hline
\end{tabular}

\subsection{Experimental Results}

The proposed approach has been tested using real data obtained with a Kinect sensor. The algorithm was implemented using $\mathrm{C}++$ and it was compared with the PCL RANSAC algorithm.

Table 3. Comparison between RANSAC algorithm and the proposed algorithm. The $\theta$ value, represents the mean angle between the normal of the reference plane and the normal of the detected plane. The value $D$ represent the RMS distance $d_{r}-d$, where $d_{r}$ and $d$ represent the distance of the of the plane from the origin, for the reference plane and the estimated plane respectively.

\begin{tabular}{||c||c|c||c|c||}
\hline $\begin{array}{c}\text { Point } \\
\text { cloud }\end{array}$ & $\begin{array}{c}\text { RANSAC } \\
\text { time(sec) }\end{array}$ & $\begin{array}{c}\text { Proposed approach } \\
\text { time(sec) }\end{array}$ & $\begin{array}{c}\theta \\
\text { (degrees) }\end{array}$ & $\begin{array}{c}D \\
\text { (meters) }\end{array}$ \\
\hline 1 & 0.269000 & 0.065380 & 3.065944 & 0.132760 \\
2 & 0.268760 & 0.078860 & 3.131493 & 0.036680 \\
3 & 0.267880 & 0.088840 & 2.727488 & 0.016781 \\
4 & 0.218440 & 0.181060 & 3.108425 & 0.018580 \\
\hline
\end{tabular}

The results of the experiment are shown in Table 3, these were obtained by running 1,000 times the algorithms. From these results, we can notice that the proposed approach has better processing time, and it provides almost the same RANSAC results. 


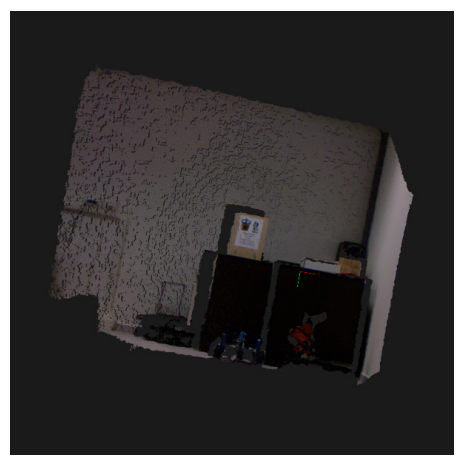

(a) Point cloud before detection

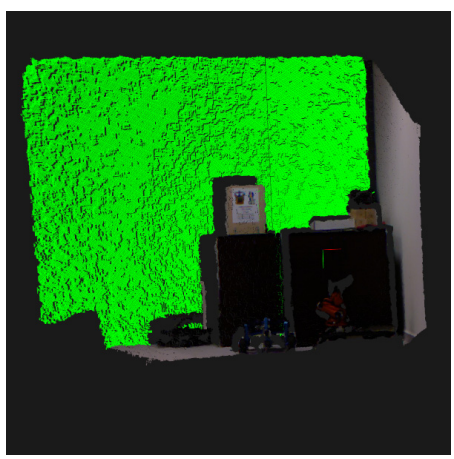

(b) Points cloud after detection

\section{Conclusions}

In this paper, we introduced an algorithm for plane detection from data obtained by a RGB-D sensor. The planes were represented using CGA, which is a powerful framework for geometric object representation in addition with its operations. The use of this framework in conjunction with PSO opens new ways for the detection of geometric objects. The experimental results show that the proposed approach is robust to outliers, and it has better performance than RANSAC.

\section{References}

1. Dorigo, M., Maniezzo, V., Colorni, A.: Ant system: optimization by a colony of cooperating agents. IEEE Transactions on Systems, Man, and Cybernetics, Part B: Cybernetics 26(1), 29-41 (1996)

2. Elbeltagi, E., Hegazy, T., Grierson, D.: Comparison among five evolutionary-based optimization algorithms. Advanced Engineering Informatics 19(1), 43 (2005)

3. Eusuff, M., Lansey, K.: Optimization of water distribution network design using the shuffled frog leaping algorithm. Journal of Water Resources Planning and Management 129(3), 210-225 (2003)

4. Hestenes, D.: New Foundations for Classical Mechanics. Kluwer (1999)

5. Hestenes, D., Li, H., Rockwood, A.: New algebraic tools for classical geometry. In: Sommer, G. (ed.) Geometric Computing with Clifford Algebra, vol. 24, pp. 3-26. Springer, Heidelberg (2001)

6. Holland, J.H.: Adaptation in Natural and Artificial Systems. MIT Press, Cambridge (1992)

7. Kennedy, J., Eberhart, R.: Particle swarm optimization. In: Proceedings of the IEEE International Conference on Neural Networks, vol. 4, pp. 1942-1948. IEEE (November 1995)

8. Li, H., Hestenes, D.: Generalized homogeneous coordinates for computational geometry. In: Sommer, G. (ed.) Geometric Computing with Clifford Algebra, vol. 24, pp. 27-60. Springer, Heidelberg (2001)

9. Merz, P., Freisleben, B.: A genetic local search approach to the quadratic assignment problem. In: in Proceedings of the 7th International Conference on Genetic Algorithms, pp. 465-472. Morgan Kaufmann (1997)

10. Parsopoulos, K., Vrahatis, M.: Particle Swarm Optimization and Intelligence Advances and Applications. IGI global (2010) 